\title{
A TCAD-based Methodology to Model the Site-Binding Charge at ISFET/Electrolyte Interfaces
}

\author{
Andrea Bandiziol, Pierpaolo Palestri, Federico Pittino, David Esseni and Luca Selmi
}

\begin{abstract}
We propose a new approach to describe in commercial TCAD the chemical reactions that occur at dielectric/electrolyte interface and make the ISFET sensitive to $p H$. The accuracy of the proposed method is successfully verified against available experimental data. We demonstrate the usefulness of the method by performing, for the first time in a commercial TCAD environment, a full two-dimensional analysis of ISFET operation, and a comparison between threshold voltage and drain current differential sensitivities in the linear and saturation regimes. The method paves the way to accurate and efficient ISFET modeling with standard TCAD tools.
\end{abstract}

Index Terms-ISFET, TCAD, Modeling, Surface Reactions.

\section{INTRODUCTION}

$\mathbf{N}$ ANOELECTRONIC biosensors enable low-cost massively parallel detection of reaction by-products and analytes with unprecedented sensitivity [1], [2]. Among many sensor devices, the Ion Sensitive Field Effect Transistor (ISFET, [3], [4], [5]) has recently attracted renewed interest for its compatibility with standard CMOS technology [6], [7], [8], [9]. ISFET-based integrated circuits have been developed for DNA sequencing [10] and for quantitative and digital Polymerase Chain Reaction (PCR) [11]. For instance, the Ion Torrent ${ }^{\mathrm{TM}}$ platform has been demonstrated down to the 110 nm CMOS technology node [12] and its further scalability has been investigated theoretically [13].

Until now ISFET modeling has mostly relied on analytical one-dimensional $V_{T}$ calculations [14], [15], [16], [17], ad-hoc simulation programs [18], [19], [20], [21] or general purpose multi-physics platforms [22], [23], [24], [25]. Now that the ISFET has become the mainstream device of a few CMOSbased sensing platforms, accurate and versatile numerical device simulations in an integrated TCAD environment are desirable to support ISFET design, extract the ISFET equivalent circuit parameters and perform mixed device-circuit analysis. Unfortunately, the commercial TCAD of most widespread use [26], [27], [28] is not equipped with models for the complex and material-dependent electrochemical processes that govern ISFET operation [29], [30], [31].

This paper extends previous investigations [32], [33], [34], [35], [36] aimed at developing a commercial TCAD based methodology for the simulation of electronic biosensors. In particular, we present a strategy to describe in TCAD the

The authors are with DIEGM, Universitá degli Studi di Udine, Via delle Scienze 208, 33100, Udine, Italy

Corresponding author: Pierpaolo Palestri, palestri@uniud.it

The research leading to this work was partially funded by the European Commission FP7 project NANOFUNCTION via the IUNET consortium.

Manuscript received ... chemical reactions at the ISFET oxide/electrolyte interface, which were not addressed previously, but are the main contribution to the ISFET threshold voltage sensitivity to $p H$. For demonstration purposes we adopt as vehicle TCAD the SDevice framework [28]; other platforms have similar limitations [26], [27], so the interest for the proposed approach extends to a broad community of TCAD users.

The paper proceeds as follows: the modeling framework and its verification against existing data is described in Section II. Simulation results about the sensitivity of short channel ISFETs featuring different gate dielectrics in the linear and the saturation regime are reported in Section III. Conclusions are summarized in Section IV.

\section{TCAD MODELS AND METHODS}

\section{A. Electrolyte Model}

Fig. 1 sketches the structure of an ISFET, where a fluid electrolyte replaces the gate of a conventional MOSFET.

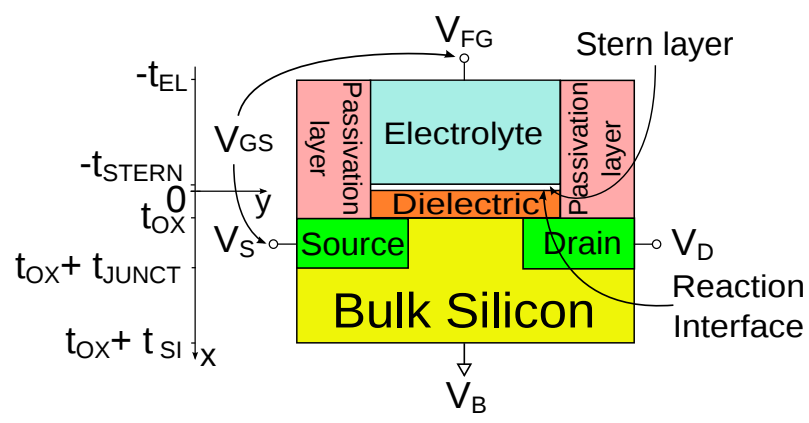

Fig. 1. Sketch of an ISFET device. The y-axis runs along the source-to-drain direction, whereas the $\mathrm{x}$-axis is normal to the channel/dielectric interface. $t_{S T E R N}$ denotes the thickness of the Stern layer, $t_{O X}, t_{J U N C T}, t_{S I}$, $t_{E L}$ the thicknesses of the dielectric, S/D junctions, substrate and electrolyte, respectively.

We model the electrolyte as described in [35], that is: we exploit the similarity between the equations for cations and anions in the electrolyte at equilibrium and those for holes and electrons in a semiconductor. A monovalent symmetric (1:1) electrolyte is thus described as an undoped semiconductor with a zero band-gap (hence: $n \cdot p=N_{C} N_{V}$ ), a constant permittivity $\left(\epsilon_{e l} \approx 80 \epsilon_{0}\right)$ and an effective density of states

$N_{C}=N_{V}=\left\{\begin{array}{l}10^{-3} \cdot N_{a v}\left(c_{0}+c H_{B}\right) \quad \text { for } \mathrm{pH}_{\mathrm{B}} \leq 7 \\ 10^{-3} \cdot N_{a v}\left(c_{0}+10^{-14} / c H_{B}\right) \text { for } \mathrm{pH}_{\mathrm{B}}>7\end{array}\right.$

where $N_{C}$ and $N_{V}$ are expressed in $\mathrm{cm}^{-3}, N_{a v}=6.02214 \cdot 10^{23}$ $\mathrm{mol}^{-1}$ is Avogadro's number, $c_{0}$ the salt ion molar concentration $(\mathrm{M}=\mathrm{mol} / \mathrm{l})$ in the bulk of the solution and 
$c H_{B}=\left[H_{B}^{+}\right]=10^{-p H_{B}}$ is the hydrogen concentration in the bulk of the solution normalized to $1 \mathrm{M}$. As an example, for $c_{0}=1 \mathrm{mM}$ and $p H_{B}=3$, we have $N_{C}=N_{V}=1.2 \cdot 10^{18} \mathrm{~cm}^{-3}$. The bulk electrolyte is defined as the region with constant potential and a zero net charge density as a result of equal total concentrations of positive and negative ions, respectively represented by $p$ and $n$; hence $n_{B}=p_{B}=N_{C}=N_{V}$.

As shown in [35], provided all ions are monovalent and their mobility and diffusivity are not too different, an equivalent symmetric 1:1 electrolyte can be defined to mimic with good accuracy the electrostatic response of complex multiion solutions. Bivalent $\mathrm{Mg}$ and $\mathrm{Ca}$ ions are sometimes used in buffer solutions (e.g. for cell culture), but typically have much smaller concentration than that of monovalent ones $(\mathrm{Na}, \mathrm{K}$, $\mathrm{Cl}$, etc.) [37]. Within these limits the proposed approach is thus applicable also to realistic electrolytes.

\section{B. Site Binding Model}

Chemical reactions at the interface between the ISFET gate dielectric and the electrolyte generate a surface charge density [29], [38], [39], [14], [40], [15] which makes the ISFET sensitive to $p H$. As a first step toward the development of a general methodology, we implement the default formulation of the well known site-binding model [29] of the chemical reactions at the amphoteric silanol $(\mathrm{SiOH})$ groups. These reactions generate a surface charge density [29], [5]

$$
\sigma_{0}\left(\psi_{0}\right)=q N_{\text {sil }}\left[\frac{c H_{S}^{2}-K_{a} K_{b}}{c H_{S}^{2}+K_{b} c H_{S}+K_{a} K_{b}}\right],
$$

where $q$ is the electron charge, $N_{s i l}$ is the number of amphoteric silanol surface sites per unit area, $c H_{S}=10^{-p H_{S}}$ is the surface $H^{+}$concentration, $K_{a}=\nu_{S i O^{-}} \cdot c H_{S} / \nu_{S i O H}$ and $K_{b}=\nu_{\mathrm{SiOH}} \cdot c H_{S} / \nu_{\mathrm{SiOH}_{2}^{+}}$are the surface dissociation constants [5], where $\nu_{\mathrm{SiO}},, \nu_{\mathrm{SiOH}}$ and $\nu_{\mathrm{SiOH}}{ }_{2}$ are the number of negative, neutral and positive silanol surface sites per unit area, respectively. Under equilibrium conditions, as it is most often the case in the electrolyte of an ISFET, and neglecting steric effects which are relevant only well above physiological salt concentrations $\left(c_{0} \gg 100 \mathrm{mM}\right)$, the surface and bulk concentrations of all monovalent ions are related by the same Boltzmann factor. For $\mathrm{H}^{+}$ions, this relation reads

$$
c H_{S}=c H_{B} \exp \left(-\frac{q \psi_{0}}{k T}\right),
$$

where $\psi_{0}$ is the potential difference between the interface and the bulk of the solution.

Equations 2 and 3 are also valid for dielectrics other than $\mathrm{SiO}_{2}$ where a metal $(M)$ replaces Silicon, and amphoteric sites are generated by $\mathrm{MOH}$ groups. This category comprises most of the dielectrics used in the semiconductor technology, such as $\mathrm{HfO}_{2}, \mathrm{Al}_{2} \mathrm{O}_{3}$ and $\mathrm{Ta}_{2} \mathrm{O}_{5}$, with the notable exception of $\mathrm{Si}_{3} \mathrm{~N}_{4}$. In fact, besides the $\mathrm{SiOH}$ groups formed by oxidation of Silicon, the $\mathrm{Si}_{3} \mathrm{~N}_{4}$ surface is characterized by additional basic sites formed by primary amine groups [41]. Dielectrics such as $\mathrm{Si}_{3} \mathrm{~N}_{4}$ and $\mathrm{Al}_{2} \mathrm{O}_{3}$ are often preferred because they have better sensitivity, and yield more stable characteristics thanks to the reduced ion diffusivity [42], [43], [44], [45], [46].
A model for the $\mathrm{Si}_{3} \mathrm{~N}_{4} /$ electrolyte interface has been proposed in [41] under the assumption that silanol and amine sites behave independently from each other. The expression for the surface charge density then reads [41], [16]:

$$
\begin{array}{r}
\sigma_{0}\left(\psi_{0}\right)=q N_{\text {sil }}\left(\frac{c H_{S}^{2}-K_{a} K_{b}}{c H_{S}^{2}+K_{b} c H_{S}+K_{a} K_{b}}\right)+ \\
+q N_{n}\left(\frac{c H_{S}}{c H_{S}+K_{n}}\right),
\end{array}
$$

where $N_{n}$ is the number of amine surface sites per unit area and $K_{n}$ the corresponding surface dissociation constant. Eqs. 2, 3 and 4 express the surface charge as a function of the local surface potential, $\psi_{0}$.

\section{TCAD implementation of the site-binding model}

Commercial TCAD allows users to introduce bias dependent surface charges in the form of interface donor or acceptor traps. The charge corresponding to a surface trap density of $N_{S}^{a}$ acceptor and $N_{S}^{d}$ donor traps per unit area reads

$$
\sigma_{T}=q\left(N_{S}^{d} f^{d}-N_{S}^{a} f^{a}\right)
$$

where $f^{d}$ and $f^{a}$ are the occupation probabilities for donor and acceptor traps, respectively. The occupation probability for an acceptor trap is expressed as [28]

$$
f^{a}=\frac{c_{C}^{a}+c_{V}^{a}}{c_{C}^{a}+c_{V}^{a}+e_{C}^{a}+e_{V}^{a}},
$$

where $c_{C}^{a}$ and $c_{V}^{a}$ are the capture rates from the conduction and valence band, respectively, and $e_{C}^{a}$ and $e_{V}^{a}$ are the emission rates to the conduction and valence band, respectively. A similar expression holds for $f_{d}$ [28].

We exploit this feature of TCAD to introduce the sitebinding charge in the simulations self-consistently. Since the sites have amphoteric behaviour and $\sigma_{0}$ can be either positive or negative depending on $p H_{B}$ and $\psi_{0}$, both acceptor and donor traps are necessary to map Eq. 2 into Eq. 5. In the following we analyze acceptor traps; similar considerations apply to donor traps.

SDevice TCAD allows the user to redefine $c_{C}^{a}, c_{V}^{a}, e_{C}^{a}$ and $e_{V}^{a}$ as arbitrary functions of the local carrier concentrations, but it is not possible to use $\psi_{0}$ as an independent variable, as prescribed by Eqs. 2 and 4. To overcome this difficulty and without loss of generality, we first make the assumption that acceptor (donor) traps exchange carriers only with the conduction (valence) band of the semiconductor representing the electrolyte, respectively; hence, we set $c_{C}^{d}=e_{C}^{d}=c_{V}^{a}=e_{V}^{a}=0$. We also note that (aside from a sign change) the Boltzmann factor in Eq.3 is the same for all monovalent anions (cations), and thus for the total anion (cation) concentration represented by the electron (hole) concentration. In formulae: $n_{S}=n_{B} \exp \left(\psi_{0} / V_{t h}\right)$ and $p_{S}=p_{B} \exp \left(-\psi_{0} / V_{t h}\right)$, where $V_{t h}=k_{B} T / q$. Therefore, by reminding that $n_{S} p_{S}=n_{B} p_{B}$ and that $n_{B}=p_{B}$, we obtain

$$
\exp \left(-\psi_{0} / V_{t h}\right)=p_{S} / \sqrt{n_{S} p_{S}}=p_{S} / \sqrt{N_{C} N_{V}},
$$


and we can re-write Eq. 2 as

$$
\sigma_{0}=q N_{s i l} \frac{c H_{B}^{2} p_{S}-K_{a} K_{b} n_{S}}{K_{a} K_{b} n_{S}+K_{b} c H_{B} \sqrt{p_{S} n_{S}}+c H_{B}^{2} p_{S}} .
$$

Eq. 8 eliminates the explicit dependence of $\sigma_{0}$ on $\psi_{0}$ in Eq. 2 . Following a similar reasoning, it is straightforward to cast also Eq. 4 in the form of a function of $n_{S}$ and $p_{S}$ instead of $\psi_{0}$. Unfortunately, the default expressions for emission and capture rates in the TCAD do not allow us to match the $n_{S}$ and $p_{S}$ dependencies of Eq. 5 to those of Eq. 8.

To overcome this difficulty we then used the Physical Model Interface (PMI) [28], that allows the user to rewrite the emission and capture rates as arbitrary functions of electric field, temperature, electron and hole concentrations at the point where the traps are introduced. By using donor and acceptor trap densities $N_{S}^{d}=N_{S}^{a}=N_{\text {sil }}$ and by assigning to the emission and capture rates the expressions

$$
\begin{gathered}
c_{V}^{d}=c H_{B}^{2} p_{S}, \\
e_{V}^{d}=K_{a} K_{b} n_{S}+K_{b} c H_{B} \sqrt{n_{S} p_{S}} \\
c_{C}^{a}=K_{a} K_{b} n_{S} \\
e_{C}^{a}=K_{b} c H_{B} \sqrt{n_{S} p_{S}}+c H_{B}^{2} p_{S},
\end{gathered}
$$

we obtain the same dependence of $\sigma_{T}$ on $p H_{B}$ and $\psi_{0}$ given by Eq. 8; hence, by Eq. 2 as well. Eq. 4 is also amenable to this TCAD implementation by introducing additional donor traps with area density $N_{n}$, and valence band capture and emission rates easily derived from Eq. 4 itself. The parameters $K_{a}$ and $K_{b}$ are easily derived from literature data (e.g. Table II) for many gate dielectrics commonly used in microelectronis, whereas $\mathrm{cH}_{B}$ is the hydrogen concentration in the bulk of the solution.

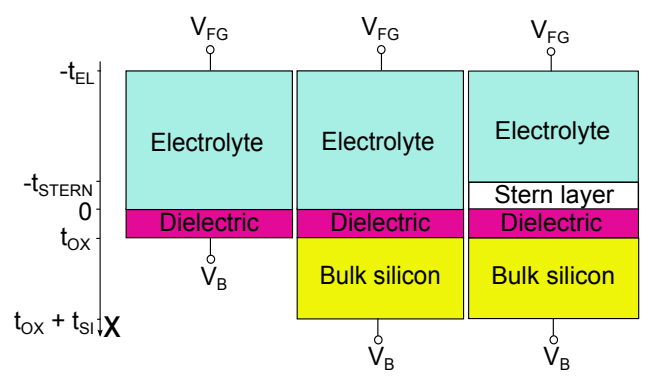

Fig. 2. Sketch of the 1D structure (left) used to validate the PMI implementation of the site-binding model and of a 1D electrolyte/insulator/semiconductor device (center). Right: Same as (center) with the Stern layer.

Fig. 3 compares Eq. 2 with its TCAD implementation for the same set of $\mathrm{SiO}_{2}$ parameters (Tabs. I, II) for the structure on the left of Fig. 2. The $\psi_{0}$ in Eq. 2 is extracted at each $V_{B}$ from the TCAD simulations. An excellent agreement is observed confirming the validity of the proposed approach.

\section{Model of the Stern Layer}

Ions in the electrolyte cannot approach the dielectric arbitrarily close, due to their finite ionic radius, and this results in a number of steric effects [47]. The electrolyte region next to the

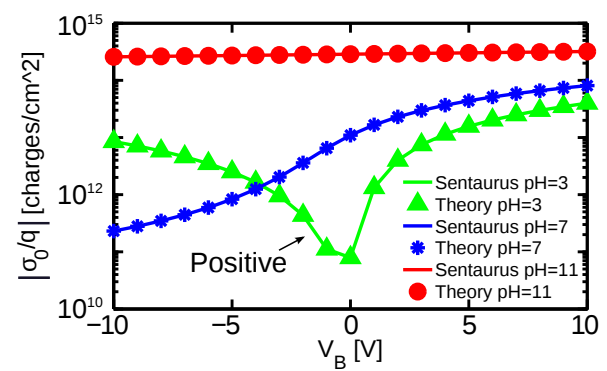

Fig. 3. Comparison between the PMI model (solid lines) and Eq. 2 (symbols) for different $V_{B}, p H$ and the same set of model parameters. The charge density is always negative unless otherwise specified. $V_{F G}=0$ $\mathrm{V}, N_{\text {sil }}=5 \times 10^{14} \mathrm{~cm}^{-2}, c_{0}=1 \mathrm{mM}, \mathrm{SiO}_{2}$ gate dielectric.

surface where the net ionic charge is essentially null is called Stern layer [48], and it is usually modeled as a thin dielectric. Since for the dielectrics of interest the interface is most often negatively charged at high $p H$ (Fig.3), the thickness $\left(t_{\text {Stern }}\right)$ is typically assumed equal to the cation's atomic radius [49] and the dielectric constant is set to a value such that the Stern layer capacitance is approximately $20 \mu \mathrm{F} / \mathrm{cm}^{2}$ [30]. A reasonable choice of $t_{S t e r n}$ appreciably mitigates the inaccuracies due to the lack of specific models for steric effects [35], [34]. For all these reasons it is highly desirable to include the Stern layer in the ISFET model and adjust its capacitance to best fit the experiments. However, if traps as those necessary to mimic the site-binding reactions are located in between the Stern layer and the gate dielectric, then $n=p=0$ regardless of the ion concentration in the electrolyte. Consequently the capture and emission rates are zero and the trapped charge is not controlled by the $p H$.

To overcome this difficulty, differently from past literature we model the Stern layer as a thin semiconductor film with a low $N_{C}=N_{V}$ corresponding to a $1 \mathrm{fM}$ solution. Consequently the Stern layer has a negligible free charge and essentially behaves as a dielectric but, at the same time, it is still possible to define traps at its interface with the actual gate dielectric. We found this workaround quite robust provided the Stern layer is not too thin. In fact, although $n_{S}$ and $p_{S}$ are very small inside the Stern layer, TCAD can still calculate the correct trap occupation function and $\sigma_{T}$ because the Boltzmann factor depends on the ratio between the carrier concentrations and the corresponding equilibrium values (Eq. 7), which are both very small in the Stern layer.

\section{E. Model Validation}

In order to check the validity of our model and to demonstrate the importance of the Stern layer, we extracted the threshold voltage $V_{T}$ versus $p H_{B}$ curve for the structures reported at the center and right of Fig. 2, and compared the results with the experimental data in [30]. To this end, $V_{T}$ is defined as the $V_{F G}$ at which the minority carrier concentration at the silicon interface $(n)$ is equal to the doping concentration in the substrate $\left(N_{A}\right)$ for $V_{B}=0 \mathrm{~V}$. The structure and the surface reaction parameters used in the paper are reported in Tabs. I and II. In general terms the parameters are extracted by fitting the model to experimental $V_{T}$ or surface potential versus $p H$ 

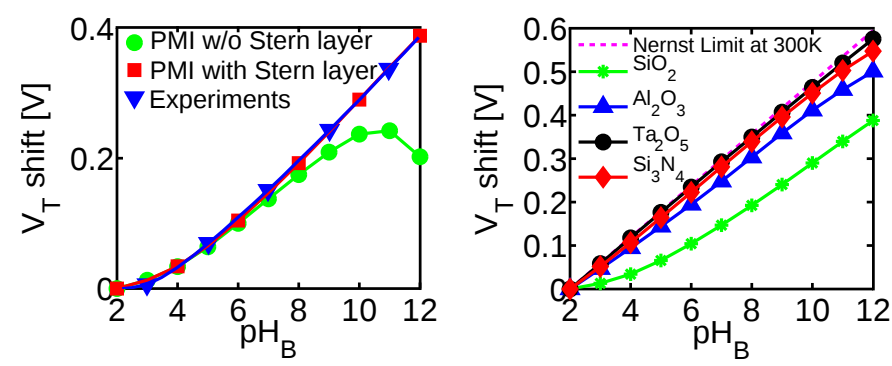

Fig. 4. Left: comparison between the TCAD simulations for the structure at the center of Fig. 2 with $\mathrm{SiO}_{2}$ dielectric (o), the SDevice simulations of the structure on the right of Fig. 2 (square) and the experimental data of [30] $(\triangle)$. Right: $V_{T}$ versus $p H_{B}$ characteristics for different dielectric materials for the structure on the right of Fig. 2 at $1 \mathrm{mM}$ salt concentration. $V_{B}=0 \mathrm{~V}$.

and ion concentration (see also Fig.5 in the following).

The results for an ISFET with $\mathrm{SiO}_{2}$ dielectric are shown in Fig. 4 and illustrate the importance of the Stern layer in order to reproduce the experiments at high $p H$. This is because when the cations accumulate at the minimum distance from the dielectric/electrolyte interface a voltage drop develops across the Stern layer which explains the observed $V_{T}$ increase. Without Stern layer, instead, at high $p H$ the ions in the electrolyte screen the surface charge resulting in a weaker $V_{T}$ increase and even a roll-off when $p H$ becomes larger than 10 .

The left plot in Fig. 4 reports the $V_{T}$ shift versus $p H$ for a few dielectrics of common use in microelectronics. The slope of these curves is the threshold voltage sensitivity $S_{V_{T}}=d V_{T} / d p H_{B}$. In agreement with the experiments in [50], [16], [30], [41], $\mathrm{Si}_{3} \mathrm{~N}_{4}, \mathrm{Ta}_{2} \mathrm{O}_{5}$ and $\mathrm{Al}_{2} \mathrm{O}_{3}$ yield $S_{V_{T}}$ values close to the theoretical $59 \mathrm{mV} / \mathrm{pH}$ Nernst limit (not shown), whereas the average sensitivity of $\mathrm{SiO}_{2}$ is lower $(\approx 40 \mathrm{mV} / \mathrm{pH})$ especially for low $p H_{B}$ values.

\begin{tabular}{||l|c|c||l|c|c||}
\hline \hline & Value & Units & & Value & Units \\
\hline \hline$t_{e l}$ & $10^{-6}$ & $\mathrm{~m}$ & $\epsilon_{e l}$ & 80 & 1 \\
\hline$t_{\text {Stern }}$ & $1.1 \cdot 10^{-10}$ & $\mathrm{~m}$ & $\epsilon_{\text {Stern }}$ & 2.5 & 1 \\
\hline$t_{\text {ox }}$ & $3 \cdot 10^{-9}$ & $\mathrm{~m}$ & $\epsilon_{\text {ox }}$ & 3.9 & 1 \\
\hline$T$ & 300 & $\mathrm{~K}$ & $N_{A}$ & $10^{17}$ & $\mathrm{~cm}^{-3}$ \\
\hline$c_{0}$ & $10^{-3}$ & $\mathrm{~mol} / 1$ & $\epsilon_{0}$ & $8.85 \cdot 10^{-14}$ & $\mathrm{~F} / \mathrm{cm}$ \\
\hline \hline
\end{tabular}

TABLE I

PARAMETER VAlues of THE STRUCTURes IN Fig. 2.

\begin{tabular}{|l|c|c|c|c|c|c|}
\hline Material & $\epsilon_{\text {rel }}$ & $N_{\text {sil }}$ & $K_{a}$ & $K_{b}$ & $N_{n}$ & $K_{n}$ \\
\hline & & {$\left[\mathrm{cm}^{-2}\right]$} & & & {$\left[\mathrm{cm}^{-2}\right]$} & \\
\hline $\mathrm{SiO}_{2}$ & 3.9 & $5 \cdot 10^{14}$ & $10^{-6}$ & $10^{2}$ & 0 & 0 \\
\hline $\mathrm{Al}_{2} \mathrm{O}_{3}$ & 14 & $8 \cdot 10^{14}$ & $10^{-10}$ & $10^{-6}$ & 0 & 0 \\
\hline $\mathrm{Ta}_{2} \mathrm{O}_{5}$ & 22 & $10^{15}$ & $10^{-4}$ & $10^{-2}$ & 0 & 0 \\
\hline $\mathrm{Si}_{3} \mathrm{~N}_{4}$ & 7.5 & $3 \cdot 10^{14}$ & $10^{-6}$ & $10^{2}$ & $2 \cdot 10^{14}$ & $10^{-10}$ \\
\hline
\end{tabular}

TABLE II

VALUES OF THE SURFACE DISSOCIATION PARAMETERS USED IN SIMULATIONS. $\mathrm{SIO}_{2}$ AND $\mathrm{AL}_{2} \mathrm{O}_{3}$ FROM [50], [30]; $\mathrm{TA}_{2} \mathrm{O}_{5}$ FROM [30]; $\mathrm{SI}_{3} \mathrm{~N}_{4}$ FROM [16].

\section{F. High salt concentration effects}

As pointed out in [31], the site-binding theory in the form previously discussed becomes inaccurate at high salt

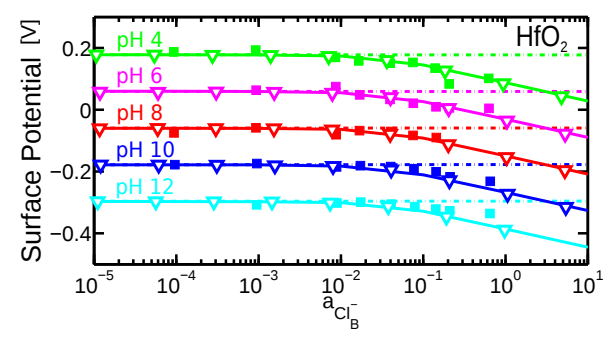

Fig. 5. Surface potential experimental data in [31] (squares) compared to the site-binding model (dash-dotted lines), the model of [31] (open triangles) and our TCAD implementation (solid lines). $a_{\mathrm{Cl}^{-}}$denotes the chlorine ion activity in the bulk of the solution. Following ref. [31], we compute the surface potential as $V_{T, P Z C}-V_{T}$, where $V_{T, P Z C}$ is the $V_{T}$ for the $p H$ of zero charge $\left(p H_{P Z C}=-0.5 \ln K_{a} \cdot K_{b}\right)$ at very low electrolyte concentration $(10 \mu \mathrm{M}) . K_{a}=K_{b}=10^{-7}, K_{c}=3.3 \cdot 10^{-6}, N_{s i l}=10^{15} \mathrm{~cm}^{-2}$.

concentration. This discrepancy is clearly visible in Fig. 5 that compares the surface potential predicted by the conventional site-binding model to the experimental data from [31]. The discrepancy has been attributed to surface complexation reactions with the chlorine ions of the electrolyte salt (typically $\mathrm{NaCl}$ or $\mathrm{KCl}$ ) of the form

$$
\mathrm{MOH}_{2}^{+}+\mathrm{Cl}_{S}^{-} \stackrel{K_{c}}{\rightleftharpoons} \mathrm{MOHCl}^{-}+\mathrm{H}_{\mathrm{B}}^{+}
$$

where $M$ is the metal of the dielectric (e.g. $\mathrm{Si}, \mathrm{Ta}, \mathrm{Al}$ or Hf). Following [31], we should thus describe the dielectric/electrolyte interface of $\mathrm{SiO}_{2}, \mathrm{HfO}_{2}, \mathrm{Al}_{2} \mathrm{O}_{3}$ and $\mathrm{Ta}_{2} \mathrm{O}_{5}$ with three coupled chemical reactions. The additional dissociation constant is $K_{c}=\nu_{\mathrm{MOHCl}^{-}} \cdot c \mathrm{H}_{\mathrm{B}} /\left(\nu_{\mathrm{MOH}_{2}^{+}} \cdot c \mathrm{Cl} l_{S}\right)$, where $c C l_{S}=10^{-p C l} l_{S}$ is the chlorine free-ions surface concentration and $\nu_{\mathrm{MOHCl}^{-}}$is the density of the negatively charged surface groups. The surface charge density is then given by

$$
\sigma_{0}=q \cdot\left(\nu_{\mathrm{MOH}_{2}^{+}}-\nu_{\mathrm{MO}^{-}}-\nu_{\mathrm{MOHCl}^{-}}\right) .
$$

Using the expression

$$
N_{s i l}=\nu_{M O^{-}}+\nu_{M O H}+\nu_{M O H_{2}^{+}}+\nu_{M O H C l}{ }^{-},
$$

we derive:

$$
\sigma_{0}=q N_{s i l}\left(\frac{c H_{S}^{2} c H_{B}-K_{a} K_{b} c H_{B}-K_{c} c C l_{S} c H_{S}^{2}}{c H_{S}^{2} c H_{B}+K_{a} K_{b} c H_{B}+K_{c} c C l_{S} c H_{S}^{2}+K_{b} c H_{S} c H_{B}}\right) \text { (13) }
$$

Eq. 13 is easy to implement in the TCAD via PMI considering two acceptor and one donor traps with suitable capture and emission rates as exemplified in Sect.II-C. The extra term, $c C l_{S}$, is (see Eq.7)

$$
c C l_{S}=c C l_{B} \frac{n_{S}}{\sqrt{n_{S} p_{S}}}=c C l_{B} \exp \left(\psi_{0} / V_{t h}\right) .
$$

Eq. 14 entails that the centroid of the bound chlorine ions charge (the so called inner Helmholtz plane) coincides with the dielectric/electrolyte interface where protonation/deprotonation reactions occur. This is a reasonable approximation that is justified by the good agreement with experiments shown in the following Section III.

The complete expression for $\sigma_{0}$ as a function of $n_{S}$ and $p_{S}$ is then given by Eq. 17 which extends Eq.8 to high chlorine concentrations. Note that for negligible $c C l_{B}$ Eq. 17 yields back Eq.8, as it should be. The model parameters of this 


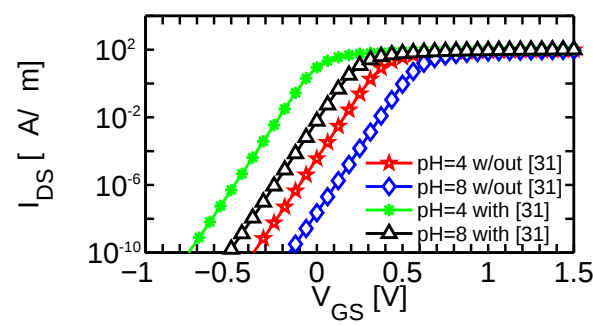

Fig. 6. $I_{D S}$ versus $V_{G S}$ characteristics of the ISFET in Fig.1 with and without the surface complexation reactions modeled as in [31]. Note the remarkable $V_{T}$ shift associated to the chlorine concentration $\left(a_{\mathrm{Cl}_{\mathrm{P}}^{-}}=c_{0}=100\right.$ $\mathrm{mM}$ ) compared to the model without surface complexation. $\mathrm{Al}_{2} \mathrm{O}_{3}$ gate dielectric.

equation either are given in the caption of Fig. $5\left(N_{s i l}, K_{a}, K_{b}\right.$ and $K_{c}$ ) or they should be computed according to the $\mathrm{pH}$ and chlorine concentration of the bulk solution $\left(\mathrm{cH}_{B}\right.$ and $\left.c \mathrm{Cl}_{B}\right)$. Fig. 5 shows that the PMI implementation of Eq. 17 (solid lines) allows the TCAD to accurately reproduce the results of [31] (symbols and dashed lines) up to high salt concentration.

Fig.6 shows how surface complexation reactions shift the devices threshold voltage to much smaller values, essentially bacause the chemical reaction in Eq.10 with $K_{c}=3.3 \cdot 10^{-6}$ increases the density of positively charged $\mathrm{MOH}_{2}^{+}$groups at the interface.

The extension to surface complexation reactions illustrates the versatility of the proposed method, which lends itself to the inclusion of many, possibly coupled, surface reactions, provided equilibrium holds in the electrolyte so that it is legitimate to substitute the Boltzmann factor for all monovalent ions with suitable ratios between the surface and bulk free carrier concentrations as in Eqs.7 and 14.

\section{RESULTS}

To demonstrate the versatility of the proposed method, we present a full two-dimensional analysis of the $V_{T}$ and $I_{D S}$ sensitivity to $p H$ changes for the planar ISFET architecture shown in Fig. 1 in the linear and saturation regions of operation. In fact, biasing the ISFET in the triode region is often the preferred choice in readout circuits designed to detect the threshold voltage sensitivity $S_{V_{T}}=\Delta V_{T} / \Delta p H_{B}$ at constant $I_{D S}$. However, several read-out circuits operate the ISFET in the saturation region, for instance when the device is part of a differential pair [6], or part of an inverter [45]. Furthermore, integrated arrays typically feature a unique fluid gate electrode for numerous ISFETs exposed to many analyte samples in parallel [10], [11]: sensing the $V_{T}$ shift of the single wires in parallel is then difficult; consequently, the sense amplifiers detect changes in the drain current at constant $V_{G S}$ and demand a large current sensitivity $S_{I_{D S}}=\Delta I_{D S} /\left[I_{D S} \Delta p H_{B}\right]$.

The dimensions of the simulated ISFET are reported in Tab. III. The source/drain doping profile decays at approxi- mately $5 \mathrm{~nm} /$ decade toward the channel region; if not otherwise stated the surface reaction model parameters are those in Tab. I. Compared to the results of the previous Section, the Stern layer thickness has been increased to $3 \AA$ while keeping the same $20 \mu \mathrm{F} / \mathrm{cm}^{2}$ capacitance [30] to facilitate numerical convergence. We verified that the current-voltage curves and the threshold voltage shifts are not affected by this choice.

\begin{tabular}{||l|c|c||l|c|c||}
\hline \hline & Value & Units & & Value & Units \\
\hline \hline$t_{\text {Stern }}$ & 3 & $\AA$ & $\epsilon_{\text {Stern }}$ & 6.82 & $\epsilon_{0}$ \\
\hline $\mathrm{t}_{\text {ox }}$ & 3 & $\mathrm{~nm}$ & $N_{A}$ & $10^{17}$ & $\mathrm{~cm}^{-3}$ \\
\hline$N_{D, \text { source }}$ & $10^{19}$ & $\mathrm{~cm}^{-3}$ & $N_{D, \text { drain }}$ & $10^{19}$ & $\mathrm{~cm}^{-3}$ \\
\hline$L_{\text {source }}$ & 200 & $\mathrm{~nm}$ & $L_{\text {drain }}$ & 200 & $\mathrm{~nm}$ \\
\hline$L_{\text {channel }}$ & 200 & $\mathrm{~nm}$ & $t_{\text {junct }}$ & 40 & $\mathrm{~nm}$ \\
\hline \hline
\end{tabular}

TABLE III

SIMULATION PARAMETERS FOR THE ISFET OF FIG. 1.

\section{A. Threshold voltage sensitivity}
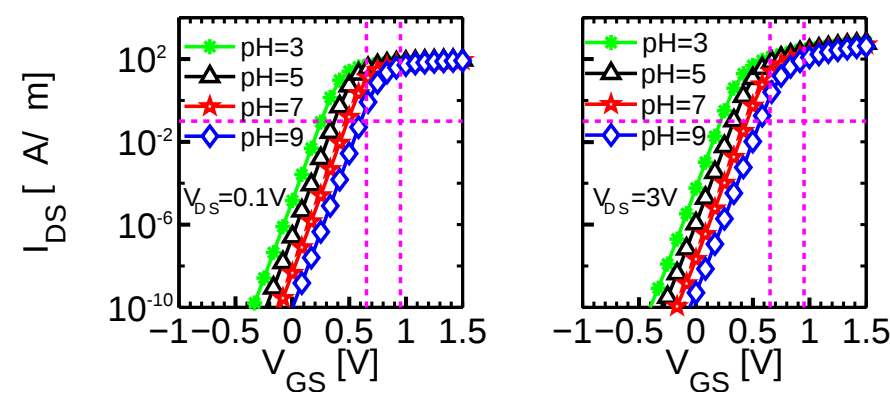

Fig. 7. Left: $I_{D S}-V_{G S}$ curves of the device in Fig. 1 with parameters from Tabs.I and III, and considering a $\mathrm{Si}_{3} \mathrm{~N}_{4}$ gate dielectric in the linear (left, $V_{D S}=0.1 \mathrm{~V}$ ) and saturation (right, $V_{D S}=3 \mathrm{~V}$ ) regions of operation. $1 \mathrm{mM}$ salt concentration. The vertical and horizontal dashed lines mark the voltage and current levels used to extract the $p H$ sensitivities.

Fig. 7 reports the $I_{D S}-V_{G S}$ curves for the device with $\mathrm{Si}_{3} \mathrm{~N}_{4}$ dielectric in the linear (left, $V_{D S}=0.1 \mathrm{~V}$ ) and saturation (right, $\left.V_{D S}=3 \mathrm{~V}\right)$ region. Due to a well-tempered device design and negligible short channel effects (subthreshold swing $S S \simeq$ 65.8 and $66.7 \mathrm{mV} / \mathrm{dec}$ at $V_{D S} \simeq 0.1 \mathrm{~V}$ and $3 \mathrm{~V}$, respectively, $\mathrm{DIBL} \simeq 13 \mathrm{mV} / \mathrm{V}$ ), the curves shift rigidly for increasing $p H$ both in the linear and saturation regimes, as we verified by checking explicitly that the $g_{m}=\left[\partial I_{D S} / \partial V_{G S}\right]$ versus $V_{G S^{-}} V_{T}$ characteristics overlap exactly.

Fig. 8 (top plot) shows the lateral potential profile at the dielectric/Stern layer interface, $\psi_{0}(y)$ and the surface potential at the channel/dielectric interface, $\psi_{s}(y)$. The surface potential decays with a short screening length $\lambda \simeq 14 \mathrm{~nm}$ due to the thin oxide and high channel doping. The lateral voltage drop remarkably modulates the negative site-binding charge (lower plot) which peaks at the source and drain side of the channel and in turn tends to equalize $\psi_{0}(y)$ and the potential at the

$$
\sigma_{0}=q N_{\text {sil }} \frac{c H_{B}^{2} p_{S}-K_{a} K_{b} n_{S}-K_{c} c C l_{B} \sqrt{n_{S} p_{S}} c H_{B}}{c H_{B}^{2} p_{S}+K_{a} K_{b} n_{S}+K_{c} c C l_{B} \sqrt{n_{S} p_{S}} c H_{B}+K_{b} \sqrt{n_{S} p_{S}} c H_{B}} .
$$



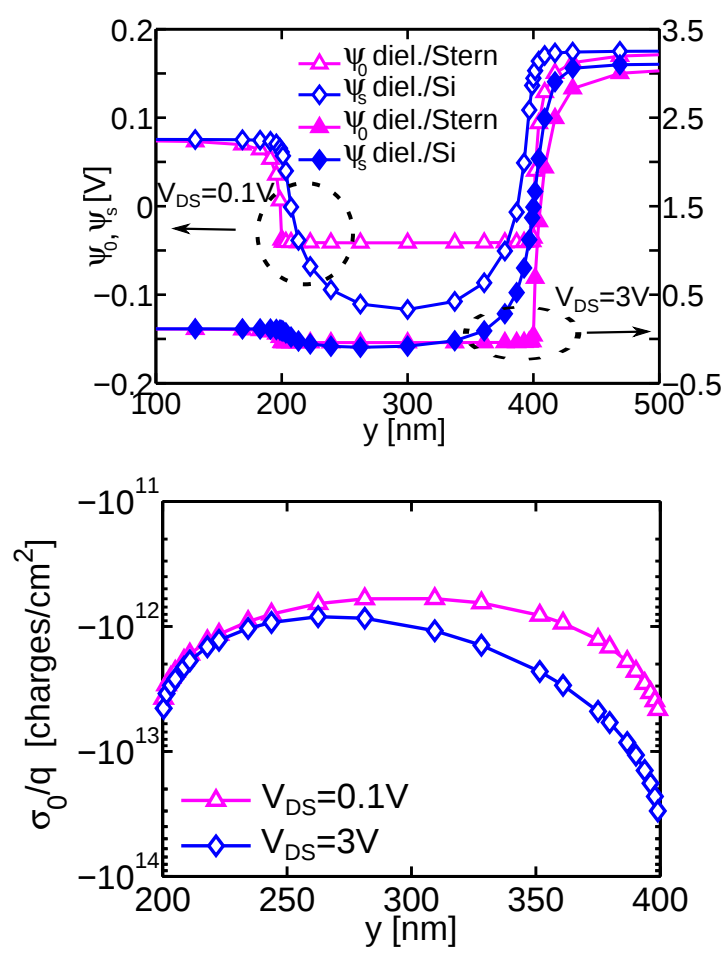

Fig. 8. Top: Potential profiles along the $y$ direction at the oxyde/electrolyte $\left(\psi_{0}\right)$ and at the silicon/oxide $\left(\psi_{s}\right)$ interfaces for a few $\mathrm{V}_{D S}$ at $\mathrm{V}_{G S}=V_{T}$ and $p H=7$. Bottom: corresponding surface charge density $\sigma_{0} . c_{0}=1 \mathrm{mM} . \mathrm{Si}_{3} \mathrm{~N}_{4}$ gate dielectric.

electrolyte/Stern layer interface (not shown). As a consequence of the almost constant $\psi_{0}(y)$ for both low and high $\mathrm{V}_{D S}$ values, the change of the $p H$ results in an amost rigid voltage shift as in Fig.7, regardless of the $\mathrm{V}_{D S}$ value.
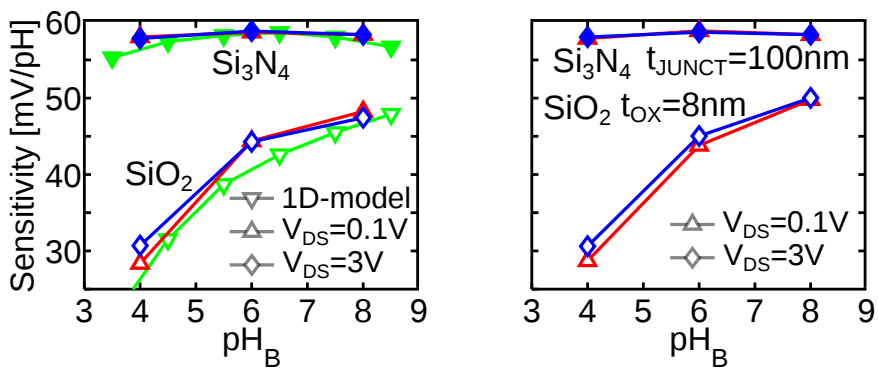

Fig. 9. Left: Differential $V_{T}$ sensitivity versus $p H$ for the ISFET with $\mathrm{Si}_{3} \mathrm{~N}_{4}$ dielectric (filled symbols) and with $\mathrm{SiO}_{2}$ dielectric (open symbols) in the linear $\left(V_{D S}=0.1 \mathrm{~V}\right)$ and saturation region $\left(V_{D S}=3 \mathrm{~V}\right)$. Calculations with the one-dimensional model [14], [15], [16], [17] (green) and with the TCAD. Right: $S_{V_{T}}$ for the same devices as in the left plot, except that the junction depth is $100 \mathrm{~nm}$ for the $\mathrm{Si}_{3} \mathrm{~N}_{4}$ device and the gate dielectric thickness is 8 nm for the $\mathrm{SiO}_{2}$ device.

In fact, Fig.9 (left plot) shows the threshold voltage sensitivity $S_{V_{T}}=\Delta V_{T} / \Delta p H_{B}$ at constant $I_{D S}=100 \mathrm{nA} / \mu \mathrm{m}$ for an ISFETs with $\mathrm{Si}_{3} \mathrm{~N}_{4}$ gate dielectric. $S_{V_{T}}$ values are almost insensitive to $p H_{B}$, and very close to the Nernst limit, as in the experiments of refs.[41], [30]. The $S_{V_{T}}$ for a device with $\mathrm{SiO}_{2}$ gate dielectric is much smaller than for $\mathrm{Si}_{3} \mathrm{~N}_{4}$, especially at low $p H_{B}$, in agreement with [50]. The results demonstrate the ability of the proposed approach to describe different ISFET technologies. We also note that the 2D TCAD model agrees well with the 1D approximation in both the linear and the saturation region, the discrepancy being in the order of only $5 \%-10 \%$. This is understood based on the discussion above, and further corroborated by the data in the right plot of Fig.9. As can be seen the threshold voltage sensitivity for devices not as well-tempered as the one in Fig.7 (because of deeper junctions, $100 \mathrm{~nm}$, or thicker gate dielectric, $8 \mathrm{~nm}$ ) is essentially the same than for the device in Fig.7.

The general purpose methodology developed here highlights that, despite the internal variables are non uniform and depend on $\mathrm{V}_{D S}$, the resulting $\mathrm{V}_{T}$ sensitivity is almost independent of $\mathrm{V}_{D S}$ and equal to the predictions of the $1 \mathrm{D}$ model.

\section{B. $I_{D S}$ sensitivity}

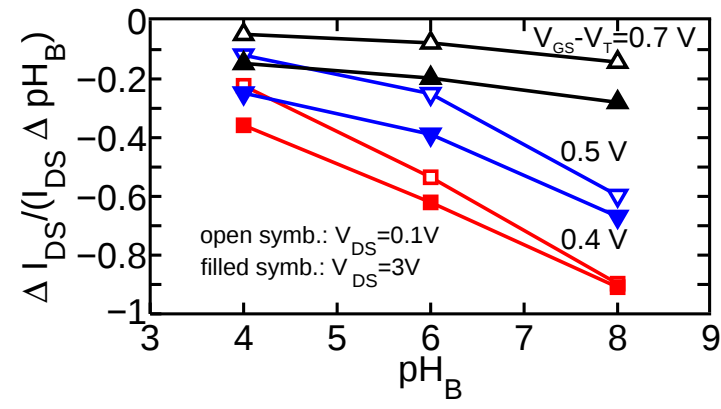

Fig. 10. Drain current sensitivity to $p H$ changes $S_{I_{D S}}$ at constant $V_{G S}$ values such that $\mathrm{V}_{G S}-\mathrm{V}_{T, p H=3}$ is the same at both $V_{D S}=0.1 \mathrm{~V}$ and $V_{D S}=3$ V. Minimum and maximum $V_{G S}$ are marked with vertical lines in Fig.7. $\mathrm{Si}_{3} \mathrm{~N}_{4}$ gate dielectric.

As already mentioned, drain current sensitivity is also relevant for applications. To investigate this aspect, Fig. 10 plots the $S_{I_{D S}}$ versus $p H_{B}$ value and for constant $V_{G S}$, corresponding to different $V_{G S^{-}} V_{T}$ depending on $p H$. The minimum and maximum $V_{G S}$ are marked by dashed vertical lines in Fig.7. The gate voltage is chosen in such a way that $\mathrm{V}_{G S}-\mathrm{V}_{T, p H=3}$ is constant. At the highest $\mathrm{V}_{G S}-\mathrm{V}_{T, p H=3}$ the ISFET operates in strong inversion for all $p H$ values. We see that the absolute value of the drain current sensitivity at constant $V_{G S}$ increases at larger $p H$, and it varies with the bias point, being larger in the saturation region than in the triode region, and at small gate overdrive. In fact, $S_{I_{D S}}=S_{V_{T}} g_{m} / I_{D S}$ and $g_{m} / I_{D S}$ increases for $\mathrm{V}_{G S}$ values approaching $\mathrm{V}_{T}$. At high $p H$, the $\mathrm{V}_{T}$ is larger than for $p H=3$, so that for the same $\mathrm{V}_{G S}$ we work closer to the threshold with larger $g_{m} / I_{D}$. This explains the large current sensitivity at large $p H$ in Fig. 10.

Working in saturation thus provides clear advantages compared to the linear region only at moderate $\mathrm{V}_{G S}-\mathrm{V}_{T}$, but no advantages in voltage sensitivity.

\section{CONCLUSIONS}

We proposed a general methodology to describe the surface charge density generated by reactions at the dielectric/electrolyte interface of ISFETs by means of commercial TCAD. By mapping the total anion and cation concentrations 
(including $\mathrm{H}^{+}$and $\mathrm{OH}^{-}$groups) into the hole and electron concentrations, we can then express the Boltzmann factor as the ratio between surface and bulk concentrations. The surface potential dependent charge is replaced by surface traps with modified concentration dependent capture and emission rates, and then solved self-consistently by the TCAD.

The method is versatile and easily applicable; many independent or coupled chemical reactions can be described provided a closed form expression can be found for $\sigma_{0}$ in terms of surface and bulk ion concentrations. The method enables full two- and three-dimensional analysis of planar, fin or nanowire ISFET device architectures in a TCAD environment. A critical re-examination of the bias dependent threshold voltage and drain current sensitivity of planar ISFETs in two dimensions has been carried out. The results highlight that a rigid shift of the $I_{D S}-V_{G S}$ characteristics versus $\mathrm{pH}$ has to be expected regardless of the operating region and, consequently, the $g_{m} / I_{D S}$ parameter rules the relation between $V_{T}$ and $I_{D S}$ sensitivities regardless of the bias point.

The model represents a significant step forward in the accurate modeling of ISFETs by means of commercial TCAD and paves the way toward the practical use of these tools in the design and optimization of integrated ISFET devices.

\section{ACKNOWLEDGMENTS}

The authors would like to thank Paolo Scarbolo for many helpful discussions.

\section{REFERENCES}

[1] M. A. Reed and N. Haven, "CMOS Biosensor Devices and Applications," in IEEE IEDM Technical Digest, 2013, pp. 208-211.

[2] A. Matsumoto and Y. Miyahara, "Current and Emerging Challenges of Field Effect Transistor Based Bio-sensing," Nanoscale, vol. 5, pp. 10 702-10718, 2013.

[3] P. Bergveld, "Development of an Ion-Sensitive Solid-State Device for Neurophysiological Measurements," IEEE Trans. on Bio-medical Engineering, no. 1, pp. 70-71, 1970.

[4] — " "Thirty years of ISFETOLOGY What happened in the past 30 years and what may happen in the next 30 years," Sensors and Actuators B: Chemical, vol. 88, no. 1, pp. 1-20, 2003.

[5] — " "ISFET, Theory and Practice," in Proceedings IEEE Sensor Conference, 2003, pp. 1-26.

[6] Y.-L. Chin, J.-C. Chou, T.-P. Sun, W.-Y. Chung, and S.-K. Hsiung, "A Novel $\mathrm{pH}$ Sensitive ISFET with on Chip Temperature Sensing Using CMOS Standard Process," Sensors and Actuators B: Chemical, vol. 76, no. $1-3$, pp. 582-593, June 2001.

[7] C. Stagni, C. Guiducci, L. Benini, B. Riccò, S. Carrara, B. Samorí, C. Paulus, M. Schienle, M. Augustyniak, and R. Thewes, "CMOS DNA Sensor Array With Integrated A / D Conversion Based on LabelFree Capacitance Measurement," IEEE Journal of Solid State Circuits, vol. 41, no. 12, pp. 2956-2964, 2006.

[8] C. Jakobson, U. Dinnar, M. Feinsod, and Y. Nemirovsky, "IonSensitive Field Effect Transistors in Standard CMOS Fabricated by PostProcessing," IEEE Sensors Jou., vol. 2, pp. 279-286, 2002.

[9] C. Jimenez-Jorquera, J. Orozco, and A. Baldi, "ISFET based microsensors for environmental monitoring." Sensors, vol. 10, no. 1, pp. 61-83, Jan. 2010.

[10] J.M. Rothberg et al., "An integrated semiconductor device enabling nonoptical genome sequencing." Nature, vol. 475, no. 7356, pp. 348-52, July 2011.

[11] C. Toumazou and et al., "Simultaneous DNA amplification and detection using a pH-sensing semiconductor system," Nature Methods, vol. 10, pp. 641-646, 2013.

[12] J. Bustillo, K. Fife, B. Merriman, and J. Rothberg, "Development of the Ion Torrent CMOS Chip for DNA Sequencing ," in IEEE IEDM Technical Digest, 2013, pp. 196-199.
[13] P. Dak, P. Nair, J. Go, and M. Alam, "Extended-gate biosensors achieve fluid stability with no loss in charge sensitivity," in Proceedings Device Research Conference, vol. 20, 2013, pp. 105-106.

[14] W. Siu and R. Cobbold, "Basic Properties of the Electrolyte- $\mathrm{SiO}_{2}-\mathrm{Si}$ System: Physical and Theoretical Aspects," IEEE Trans. on Electron Devices, no. 1, pp. 1805-1815, 1979.

[15] C. Fung, P. Cheung, and W. Ko, "A Generalized Theory of an Electrolyte-Insulator-Semiconductor Field-Effect Transistor," IEEE Trans. on Electron Devices, no. 1, 1986.

[16] M. Grattarola, G. Massobrio, and S. Martinoia, "Modeling $\mathrm{H}^{+}$-Sensitive FET's with SPICE," IEEE Trans. on Electron Devices, vol. 39, no. 4, pp. 1-6, 1992.

[17] A. Matsumoto and Y. Miyahara, "Analytical Modeling of Biotransistors," IET Circuits, Devices and Systems, vol. 2, pp. 158-165, 2008.

[18] Y.Wang and G.Li, "Performance Investigation for a Silicon Nanowire FET Biosensor Using Numerical Simulation," in Proc. IEEE Nanotechnology Materials and Devices Conference, 2010, pp. 81-86.

[19] W. R. Frensley, "Performance Analysis of Si Nanowire Biosensor by Numerical Modeling for Charge Sensing," IEEE Transactions on Nanotechnology, vol. 11, no. 3, pp. 501-512, May 2012.

[20] S. Baumgartner, C. Heitzinger, A. Vacic, and M. A. Reed, "Predictive simulations and optimization of nanowire field-effect PSA sensors including screening," Nanotechnology, vol. 24, no. 22, p. 225503, 2013. [Online]. Available: http://stacks.iop.org/0957-4484/24/i=22/a=225503

[21] F. Pittino and L. Selmi, "Use and comparative assessment of the cvfem method for poissonboltzmann and poissonnernstplanck three dimensional simulations of impedimetric nano-biosensors operated in the dc and ac small signal regimes," Computational Methods in Applied Mechanics and Engineering, pp. 902-923, 2014.

[22] K. B. Parizi, A. J. Yeh, A. S. Y. Poon, and H.-S. P. Wong, "Exceeding Nernst Limit $(59 \mathrm{mV} / \mathrm{pH})$ : CMOS-Based $\mathrm{pH}$ Sensor for Autonomous Applications," in IEEE IEDM Technical Digest, 2012, pp. 557-560.

[23] COMSOL, "Comsol Multiphysics Ref. Manual," Tech. Rep., 2013.

[24] M. Webster, I. Timoshkin, S.J.MacGregor, and M. Mattey, "Computer Aided Modeling of an Interdigitated Microelectrode Array Impedance Biosensor for the Detection of Bacteria," IEEE Trans. on Dielectrics and Electrical Insulation, vol. 16, pp. 1356-1363, 2009.

[25] Integrated Engineering Software, "ELECTRO/COULOMB Ref. Manual," Tech. Rep., 2013.

[26] Silvaco, "ATLAS user's manual," Tech. Rep., 2013.

[27] TMA, "MEDICI user's manual," Tech. Rep., 2013.

[28] Synopsys, Sentaurus Device User Guide, 2013.

[29] D. Yates, S. Levine, and T. Healy, "Site-binding model of the electrical double layer at the oxide/water interface," Journal of Chemical Society, Faraday Transactions, vol. 70, pp. 1807-1818, 1974.

[30] R. van Hal, J. Eijkel, and P. Bergveld, "A general model to describe the electrostatic potential at electrolyte oxide interfaces," Advances in Colloid and Interface Science, vol. 69, no. 1-3, pp. 31-62, Dec. 1996.

[31] A. Tarasov, M. Wipf, R. Stoop, and K. Bedner, "Understanding the electrolyte background for biochemical sensing with ion-sensitive fieldeffect transistors," ACS Nano, no. 10, pp. 9291-9298, 2012.

[32] I.-Y. Chung, H. Jang, J. Lee, H. Moon, S. M. Seo, and D. H. Kim, "Simulation Study on Discrete Charge Effects of SiNW Biosensors According to Bound Target Position Using a 3D TCAD Simulator," Nanotechnology, vol. 23, no. 6, p. 065202, 2012.

[33] D. Welch, S. Shah, S. Ozev, and J. B. Christen, "Experimental and Simulated Cycling of ISFET Electric Fields for Drift Reset," IEEE Electron Device Lett., vol. 34, pp. 456-458, 2013.

[34] P. Palestri, R. Sette, F. Pittino, F. Saccon, D. Esseni, and L. Selmi, "Simulation of nano-biosensors based on conventional TCAD," in Proceedings 14th International Conference on Ultimate Integration on Silicon (ULIS), Mar. 2013, pp. 77-80.

[35] F. Pittino, P. Palestri, P. Scarbolo, D. Esseni, and L. Selmi, "Models for the use of commercial TCAD in the analysis of silicon-based integrated biosensors," Solid-State Electronics, vol. 98, pp. 63-69, 2014.

[36] D. Passeri, A. Morozzi, K. Kanxheriand, and A. Scorzoni, "Numerical Simulation of ISFET Structures for BioSensing Devices with TCAD Tools," in Proceedings IWBBIO, 2014, pp. 425-435.

[37] F. Spiga, "Private Communication," Tech. Rep., 2014.

[38] J. Davis, R. James, and J. Leckie, "Surface ionization and complexation at the oxide/water interface. I. Computation of Electrical Double Layer Properties in Simple Electrolytes," Jou. of Colloid and Interface Science, vol. 63, no. 3, pp. 480-499, Mar. 1978.

[39] J. Drzymala, J. Lekki, and J. Laskowski, "Surface dissociation constants for solid oxide/aqueous solution systems," Colloid and Polymer Science, vol. 772, pp. 768-772, 1979. 
[40] L.J.Bousse and N. De Rooij and P. Bergveld, "The influence of counterion adsorption on the $\psi_{0} / \mathrm{pH}$ characteristics of insulator surfaces," Surface Science, vol. 135, pp. 479-496, 1983.

[41] D. Harame and L. Bousse, "Ion-sensing devices with silicon nitride and borosilicate glass insulators," IEEE Trans. on Electron Devices, no. 8, pp. 1700-1707, 1987.

[42] H. Abe, M. Esashi, and T. Matsuo, "ISFET's using inorganic gate thin films," IEEE Trans. on Electron Devices, vol. 26, pp. 1939-1944, 1979.

[43] P. Bergveld and A. Sibbald, Analytical and biomedical applications of ion-selective field effect transistors, Elsevier, Ed. Elsevier, Amsterdam, 1988, vol. 23.

[44] B. Jr., B. Dorvel, J.Go, P. Nair, O. Elibol, G. Credo, J. Daniels, E. Chow, X.Su, M.Varma, M. Alam, and R.Bashir, "High-k dielectric $\mathrm{Al}_{2} \mathrm{O}_{3}$ nanowire and nanoplate field effect sensors for improved $\mathrm{pH}$ sensing," Biomed Microdevices, vol. 13, pp. 335-344, 2011.

[45] J. Lee, S. Hwang, B. Choi, J. H. Lee, D. Moon, M. Seol, C. Kim, I. Chung, B. Park, Y. Choi, D. M. Kim, D. H. Kim, and S. Choi, "A Novel SiNW/CMOS Hybrid Biosensor for High Sensitivity/Low Noise," in IEEE IEDM Technical Digest, 2013, pp. 385-388.

[46] K. Bedner, V. A. Guzenko, A. Tarasov, M. Wipf, R. L. Stoop, D. Just, S. Rigante, W. Fu, R. A.Minamisawa, C. David, and et al., "pH Response of Silicon Nanowire Sensors: Impact of Nanowire Width and Gate Oxide," Sensors and Materials, vol. 25, no. 8, pp. 567-576, 2010.

[47] M. S. Kilic, M. Z. Bazant, and A. Ajdari, "Steric effects in the dynamics of electrolytes at large applied voltages. ii. modified Poisson-NernstPlanck equations," Phys. Rev. E, vol. 75, p. 021503, Feb 2007.

[48] O. Stern, "Zur Theorie der elektrolytischen Doppelschicht," Z. Elektrochem., vol. 30, pp. 508-516, 1924.

[49] Y. Marcus, "Ionic radii in aqueous solutions," Chemical Reviews, vol. 88, p. 147S, 1988.

[50] A. V. den Berg and P. Bergveld, "Sensitivity Control of ISFETs by Chemical Surface Modification," Sensors and Actuators B: Chemical, vol. 8, pp. 129-148, 1985. 